\title{
THE MEAN VALUE THEOREM AND ANALYTIC FUNCTIONS
}

\author{
by ELGIN H. JOHNSTON
}

(Received 20th January 1982)

It is well known that the mean value theorem (MVT) does not, in general, hold for analytic functions. The most familiar example to this effect is $f(z)=e^{z}$ since $e^{2 \pi i}-e^{0} \neq 2 \pi i e^{z_{0}}$ for any $z_{0} \in \mathbb{C}$. On the other hand, it is easy to show that the MVT holds in $\mathbb{C}$ if $f(z)$ is a polynomial of degree at most 2 . Thus it is natural to ask what conditions on a function $f(z)$ analytic in a domain $D$ are necessary and sufficient for $f(z)$ to satisfy the MVT in $D$. This is one of the questions answered in this paper.

Many authors have devised "substitutes" for the MVT that do apply to all analytic functions. For example Samuelsson [5] (see also Robertson [3] and Novinger [2]) has proved the following local version of the mean value theorem.

Theorem A. Let $f(z)$ be analytic in a domain containing $z_{0}$. Then there is a neighbourhood $N$ of $z_{0}$ such that if $z_{1}$ is any point in $N$, then there exists a point $z$ with $\left|z-\frac{1}{2}\left(z_{0}+z_{1}\right)\right|<\frac{1}{2}\left|z_{0}-z_{1}\right|$ and such that

$$
f\left(z_{1}\right)-f\left(z_{0}\right)=f^{\prime}(z)\left(z_{1}-z_{0}\right)
$$

Notice that the point $z$ does not necessarily lie on the segment $\left[z_{0}, z_{1}\right]$. McLeod [1] has proved a version of the MVT that involves a convex combination of derivatives on $\left[z_{0}, z_{1}\right]$.

Theorem B. Let $f(z)$ be analytic in a domain $D$. If $z_{0}, z_{1} \in D$ and the segment $\left[z_{0}, z_{1}\right] \subseteq D$, then there are points $w_{0}, w_{1} \in\left(z_{0}, z_{1}\right)$ and there is $a \lambda(0 \leqq \lambda \leqq 1)$ with

$$
f\left(z_{1}\right)-f\left(z_{0}\right)=\left(z_{1}-z_{0}\right)\left[\lambda f^{\prime}\left(w_{1}\right)+(1-\lambda) f^{\prime}\left(w_{0}\right)\right] \text {. }
$$

In this paper we look in a direction different from those in Theorems A and B and instead ask for what analytic functions does the classical MVT hold. We actually ask for more ... in particular when does a pair of analytic functions satisfy the generalised MVT? This is made more precise in the following definition.

Definition 1. Let $f(z)$ and $g(z)$ be functions analytic in a domain $D \subseteq \mathbb{C}$, and suppose that $g(z)$ is one-to-one in $D$. Then $f(z)$ and $g(z)$ satisfy the generalised mean value property $(G M V P)$ on $D$ if, whenever the line segment $\left[z_{1}, z_{2}\right] \subseteq D\left(z_{1} \neq z_{2}\right)$, there is a 
point $c \in\left(z_{1}, z_{2}\right)$ such that

$$
\frac{f\left(z_{2}\right)-f\left(z_{1}\right)}{g\left(z_{2}\right)-g\left(z_{1}\right)}=\frac{f^{\prime}(c)}{g^{\prime}(c)} .
$$

In the above definition, the classical MVP arises if we take $g(z)$ to be linear. Since the GMVP is clearly satisfied if $f(z) \equiv$ constant, we eliminate this case from our considerations.

Theorem 2. Let $f(z)$ ( $\not \equiv$ constant) be analytic in a domain $D$ and let $g(z)$ be analytic and one-to-one in $D$. Then the following are equivalent.

(i) $f(z)$ and $g(z)$ satisfy the GMVP on $D$.

(ii) $\frac{f^{\prime \prime \prime}(z)}{f^{\prime}(z)}=\frac{g^{\prime \prime \prime}(z)}{g^{\prime}(z)}$ and $\frac{f^{(5)}(z)}{f^{\prime}(z)}=\frac{g^{(5)}(z)}{g^{\prime}(z)}$

as meromorphic functions in $D$.

(iii) One of the following statements holds; in (a) and (c) it is assumed that $g$ is univalent in $D$ :

(a) $f(z)$ and $g(z)$ are nonconstant polynomials of degree at most 2 ,

(b) $f(z)=A g(z)+B$ for some complex constants $A$ and $B(A \neq 0)$,

(c) $f(z)=A \cos \alpha z+B \sin \alpha z+C$ and $g(z)=D \cos \alpha z+E \sin \alpha z+F$ where $A, B, C, D, E, F$ are complex constants and $(|A|+|B|)(|D|+|E|) \alpha \neq 0$.

Proof. (iii) $\Rightarrow$ (i). This is easily checked.

(i) $\Rightarrow$ (ii). Select $a \in D$ with $f^{\prime}(a) \neq 0$. Since neither the addition of constants to $f(z)$ and $g(z)$ nor the translation of the variable affects the GMVP, we may assume $a=0=f(0)=g(0)$. Since $g^{\prime}(0) \neq 0, g^{-1}(w)$ is analytic in a neighbourhood of $w=0$, with $g^{-1}(0)=0$. Thus $f \circ g^{-1}(w)$ is analytic in a neighbourhood of 0 and we can write

$$
f \circ g^{-1}(w)=A_{1} w+A_{2} w^{2}+\cdots
$$

for $|w|<\varepsilon$, where $\varepsilon$ is a suitable positive number. Letting $w=g(z)$, we find that

$$
f(z)=A_{1} g(z)+A_{2}(g(z))^{2}+\cdots
$$

for $|z|<\delta$, where $\delta$ is a sufficiently small positive number.

It is clear that the pair of functions $f(z)$ and $g(z)$ satisfy the GMVP on $D$ if and only if the pair $f(z)-k g(z)$ and $g(z)$ do $\left(k=\right.$ constant). So taking $F(z)=f(z)-A_{1} g(z)$, we may assume that $F(z)$ and $g(z)$ satisfy the GMVP on $D$.

If $F(z)$ is a constant function, then $f(z)=A_{1} g(z)+B$, and $A_{1} \neq 0$ since $f(z)$ is not identically constant. In this case it is easy to check that (ii) holds.

Suppose $F(z)$ is not constant. Since $F(0)=F^{\prime}(0)=0$ it follows that $F(z)$ takes the value 0 at $z=0$ with multiplicity $n \geqq 2$. Thus $[4$, p. 216] there is a disc $\Delta(0, \eta) \subseteq \Delta(0, \delta)$ such that $F^{\prime}(z) \neq 0$ in $\Delta^{\prime}(0, \eta)$ and there is a neighbourhood $N$ of 0 with $N \subseteq \Delta(0, \eta)$ such that $F(z)$ is an $n$-to-one mapping on $N$. In fact each $w \in F(N)-\{0\}$ is taken on at $n$ distinct points of 
$N-\{0\}$. If $n \geqq 3$, then we can find distinct points $z_{1}, z_{2}, z_{3} \in N-\{0\}$ with $F\left(z_{1}\right)=F\left(z_{2}\right)$ $=F\left(z_{3}\right)$. By the GMVP, each of the segments $\left(z_{1}, z_{2}\right),\left(z_{1}, z_{3}\right),\left(z_{2}, z_{3}\right)$ must contain a zero of $F^{\prime}(z)$. However these segments are all contained in $\Delta(0, \eta)$ and 0 is the only zero of $F^{\prime}(z)$ in $\Delta(0, \eta)$. Thus $0 \in\left(z_{1}, z_{2}\right) \cap\left(z_{2}, z_{3}\right) \cap\left(z_{1}, z_{3}\right)$. This is impossible for distinct points $z_{1}, z_{2}, z_{3}$. It follows that $n=2$. Thus $F(z)$ is a two-to-one mapping on $N$ and, if $z_{1}, z_{2}$ are distinct points of $N-\{0\}$, then

$$
F\left(z_{1}\right)=F\left(z_{2}\right) \text { implies that } 0 \in\left(z_{1}, z_{2}\right) \text {, i.e. } z_{1} / z_{2}<0 \text {. }
$$

Let $l$ be any line through 0 and let $H_{1}, H_{2}$ be the two open half planes determined by l. Let $N_{i}=H_{i} \cap N(i=1,2)$. It follows from (2) that $F(z)$ is univalent on each of $N_{1}, N_{2}$. Furthermore, for $i=1,2, F\left(N_{i}\right)=F(N)-\gamma$ where $\gamma=F(l \cap N)$ is a simple analytic arc with one endpoint 0 . We can then define analytic functions $h_{i}: F(N)-\gamma \rightarrow N_{i}(i=1,2)$ with $\left(h_{i} \circ F\right)(z)=z \quad\left(z \in N_{i}\right)$. Since $0 \notin N_{i} \quad(i=1,2)$, neither $h_{1}$ nor $h_{2}$ takes the values 0 . Thus $h_{1}(w) / h_{2}(w)$ is analytic on $F(N)-\gamma$ and by $(2), h_{1}(w) / h_{2}(w)<0$ on $F(N)-\gamma$. Thus $h_{1}(w) / h_{2}(w) \equiv k$ ( $k$ some negative constant). Now let $\tilde{l}$ be a line through 0 and assume the acute angle formed by $l$ and $\tau$ is less than $\pi / 10$. Let $\tilde{H}_{1}$ and $\tilde{H}_{2}$ be the half planes determined by $\tilde{l}$, and labeled so that $\tilde{H}_{1} \cap H_{1}$ is a sector of angle measure greater than $9 \pi / 10$. Analagous to the previous development, define $\tilde{N}_{i}=\tilde{H}_{i} \cap N(i=1,2), \tilde{\gamma}=F(N \cap \tilde{l})$ and $\tilde{h}_{i}: F(N)-\tilde{\gamma} \rightarrow \tilde{N}_{i}(i=1,2)$ with $\left(\tilde{h}_{i} \circ F\right)(z)=z\left(z \in \tilde{N}_{i}\right)$. Then

$$
\left.\tilde{h_{i}}\right|_{F\left(N_{i} \cap \tilde{N}_{i}\right)}=\left.h_{i}\right|_{F\left(N_{i} \cap \tilde{N}_{i}\right)}
$$

$(i=1,2)$, and it follows that $\tilde{h}_{1}(w) / \tilde{h}_{2}(w) \equiv k$ on $F(N)-\tilde{\gamma}$. Now let $z_{0} \in N_{1} \cap \tilde{N}_{2}$. Then there is a point $z_{0}^{\prime} \in N_{2} \cap \tilde{N}_{1}$ with $F\left(z_{0}\right)=F\left(z_{0}^{\prime}\right)$. We then have

$$
k=\frac{z_{0}}{z_{0}^{\prime}}=\frac{h_{1}\left(F\left(z_{0}\right)\right)}{h_{2}\left(F\left(z_{0}\right)\right)}=\frac{\tilde{h}_{2}\left(F\left(z_{0}\right)\right)}{\tilde{h}_{1}\left(F\left(z_{0}\right)\right)}=\frac{z_{0}^{\prime}}{z_{0}}=\frac{1}{k} .
$$

Hence $k=-1$.

From the above argument we may conclude that if $z_{1}, z_{2}$ are distinct points in $N$ with $F\left(z_{1}\right)=F\left(z_{2}\right)$, then $z_{1}=-z_{2}$. Conversely, if $z_{1}=-z_{2}$, we must have $F\left(z_{1}\right)=F\left(-z_{2}\right)=F\left(z_{2}\right)$, showing that $F(z)$ is an even function. Thus

$$
f^{\prime \prime \prime}(0)-A_{1} g^{\prime \prime \prime}(0)=F^{\prime \prime \prime}(0)=0
$$

and

$$
f^{(5)}(0)-A_{1} g^{(5)}(0)=F^{(5)}(0)=0
$$

Since $A_{1}=f^{\prime}(0) / g^{\prime}(0)$ and $a=0$ was chosen without loss of generality, we see that (ii) holds for all $z \in D$ at which $f^{\prime}(z) \neq 0$. Since $g^{\prime}(z)$ is never 0 on $D$, it follows that $f^{\prime \prime \prime}(z) / f^{\prime}(z)$ and $f^{(5)}(z) / f^{\prime}(z)$ have only removable singularities on $D$. Thus (ii) holds in $D$. (ii) $\Rightarrow$ (iii). We consider three cases 
Case $a$. If $f^{\prime \prime \prime}(z) / f^{\prime}(z) \equiv g^{\prime \prime \prime}(z) / g^{\prime}(z) \equiv 0$ on $D$, then it follows that $f$ and $g$ are polynomials of degree one or two. Observe that $f^{(5)}(z) / f^{\prime}(x)=g^{(5)}(z) / g^{\prime}(z)$ is also satisfied by such $f$ and $g$.

Case $b$. If $f^{\prime \prime \prime}(z) / f^{\prime}(z)=g^{\prime \prime \prime}(z) / g^{\prime}(z) \equiv k \neq 0$ on $D$, then it follows immediately that $f(z)=$ $A \cos \alpha z+B \sin \alpha z+C$ and $g(z)=D \cos \alpha z+E \sin \alpha z+F$ where $|A|+|B| \neq 0,|D|+|E| \neq 0$ and $\alpha^{2}=-k$. Note that $f^{(5)}(z) / f^{\prime}(z)=g^{(5)}(z) / g^{\prime}(z)$ is also true for such $f$ and $g$.

Case c. If $f^{\prime \prime \prime}(z) / f^{\prime}(z)=g^{\prime \prime \prime}(z) / g^{\prime}(z)=u(z)$ where $u(z)$ is not constant on $D$, then since $g^{\prime}(z)=0$ we see that $u(z)$ is analytic on $D$. Thus

$$
g^{\prime \prime \prime}(z)=g^{\prime}(z) u(z)
$$

and differentiation gives

$$
g^{(5)}(z)=g^{\prime \prime \prime}(z) u(z)+2 g^{\prime \prime}(z) u^{\prime}(z)+g^{\prime}(z) u^{\prime \prime}(z)
$$

Dividing by $g^{\prime}(z)$ we have

$$
\frac{g^{(5)}(z)}{g^{\prime}(z)}=(u(z))^{2}+\frac{2 g^{\prime \prime}(z)}{g^{\prime}(z)} u^{\prime}(z)+u^{\prime \prime}(z) .
$$

Similarly,

$$
\frac{f^{(5)}(z)}{f^{\prime}(z)}=(u(z))^{2}+\frac{2 f^{\prime \prime}(z)}{f^{\prime}(z)} u^{\prime}(z)+u^{\prime \prime}(z) .
$$

Since $u^{\prime}(z) \not \equiv 0$ in $D$ it follows that

$$
\frac{f^{\prime \prime}(z)}{f^{\prime}(z)}=\frac{g^{\prime \prime}(z)}{g^{\prime}(z)}
$$

for all $z \in D$. Hence

$$
\frac{d}{d z} \frac{f^{\prime}(z)}{g^{\prime}(z)} \equiv 0
$$

and so,

$$
\frac{f^{\prime}(z)}{g^{\prime}(z)} \equiv A,
$$

Thus $f^{\prime}(z)=A g^{\prime}(z)$ and $f(z)=A g(z)+B$.

As an immediate corollary we can characterise those analytic functions that satisfy the MVT on $D$. 
Corollary 3. Let $f(z)$ be analytic on a domain $D$. Then $f(z)$ satisfies the MVT on $D$ (i.e. the $G M V P$ with $g(z)=z$ ) if and only if $f(z)$ is a polynomial of degree at most 2.

Remark 4. Since the arguments in the proof of Theorem 2 were all local, it is clear that the univalence hypothesis on $g$ can be dropped. If we instead require only that $g(z)$ is not constant on $D$. then the proof of Theorem 2 shows that (ii) holds at all points where $f^{\prime}(z) g^{\prime}(z) \neq 0$. It follows that the equalities in (ii) actually hold in all of $D$ as equalities between meromorphic functions. The equation

$$
\frac{f\left(z_{1}\right)-f\left(z_{2}\right)}{g\left(z_{1}\right)-g\left(z_{2}\right)}=\frac{f^{\prime}(c)}{g^{\prime}(c)}
$$

when $g\left(z_{1}\right)=g\left(z_{2}\right)\left(z_{1} \neq z_{2}\right)$ can then be interpreted as saying that there is a point $c \in\left(z_{1}, z_{2}\right)$ such that the order of the pole of $\left(f\left(z_{1}\right)-f(z)\right) /\left(g\left(z_{1}\right)-g(z)\right)$ at $z_{2}$ is the same as the order of the pole of $f^{\prime}(z) / g^{\prime}(z)$ at $c$. Indeed this is easily checked for those functions $f(z)$ and $g(z)$ listed in (iii).

Remark 5. Although the condition $f^{(5)}(z) / f^{\prime}(z)=g^{(5)}(z) / g^{\prime}(z)$ was used in only one case of the proof of (ii) $\Rightarrow$ (iii), the condition cannot be dropped. For example, it is easy to check that if $f(z)=z^{4}$ and $g(z)=z^{-1}$ are analytic on a domain $D$, then $f^{\prime \prime \prime}(z) / f^{\prime}(z)=$ $g^{\prime \prime \prime}(z) / g^{\prime}(z)$. However $f(z)$ and $g(z)$ will not satisfy the GMVP on $D$. (Note that $f^{(5)}(z) / f^{\prime}(z) \neq g^{(5)}(z) / g^{\prime}(z)$ on $\left.D\right)$.

Remark 6. When discussing the MVT it seems appropriate to mention Rolle's Theorem. We will say that a non constant function $f(z)$ analytic on $D$ satisfies the Rolle Property (RP) on $D$ if $\left[z_{1}, z_{2}\right] \subseteq D\left(z_{1} \neq z_{2}\right)$ and $f\left(z_{1}\right)=f\left(z_{2}\right)$ imply the existence of a point $c \in\left(z_{1}, z_{2}\right)$ such that $f^{\prime}(c)=0$. It is clear that if $f^{\prime}\left(z_{0}\right) \neq 0\left(z_{0} \in D\right)$ then $f(z)$ satisfies RP in a neighbourhood of $z_{0}$. This illustrates a major difference between RP and the MVP. A function may satisfy RP locally on a domain $D$ without satisfying RP on the whole domain $D$, while if functions $f(z)$ and $g(z)$ satisfy the GMVP on any open subset of $D$, then they satisfy the GMVP on $D$. However, since only local arguments were used in the proof of Theorem 2, the same arguments can be used to prove the following result.

Theorem 7. If $f(z)$ is analytic in a domain $D, f^{\prime}\left(z_{0}\right)=0$ and $f(z)$ satisfies the RP on $D$, then $f\left(z+z_{0}\right)$ is an even function in a neighbourhood of 0 . Furthermore, if $f$ is not identically constant, then $f^{\prime \prime}\left(z_{0}\right) \neq 0$.

A somewhat longer argument can be used to prove the following more informative result.

Theorem 8. If $f(z)$ is analytic, nonconstant and satisfies $R P$ on a convex domain $D$, then $f(z)$ takes no value with multiplicity greater than two in $D$. 
Proof. Suppose $f(z)$ takes the value $a$ with multiplicity at least 3 . Then three cases might arise:

Case 1. There is a point $z_{0} \in D$ with $f\left(z_{0}\right)=a$ and $f^{\prime}\left(z_{0}\right)=f^{\prime \prime}\left(z_{0}\right)=0$.

Case 2. There are distinct points $z_{0}, z_{1} \in D$ with $f\left(z_{0}\right)=f\left(z_{1}\right)=a$ and $f^{\prime}\left(z_{0}\right)=0$.

Case 3. There are distinct points $z_{0}, z_{1}, z_{2} \in D$ with $f\left(z_{0}\right)=f\left(z_{1}\right)=f\left(z_{2}\right)=a$.

In Case 1 we arrive at a contradiction by applying Theorem 7. In Cases 2 and 3 it can be shown that $f^{\prime}(z) \equiv 0$ on $D$, contradicting the fact that $f$ is not identically constant.

If Case 2 holds, then by the Open Mapping Theorem and Theorem 7 we may find a neighbourhood $N$ of $z_{0}$ such that $f\left(z+z_{0}\right)$ is even in $N-z_{0}=\left\{z-z_{0}: z \in N\right\}$ and such that $f(z)$ is a two-to-one mapping on $N$, with $f^{\prime}(z) \neq 0$ on $N-\left\{z_{0}\right\}$. Depending on whether $f^{\prime}\left(z_{1}\right) \neq 0$ or $f^{\prime}\left(z_{1}\right)=0$ we may find a neighbourhood $N^{\prime}$ of $z_{1}$ on which $f(z)$ is a one-to-one mapping or a two-to-one mapping. In either case both $f(N)$ and $f\left(N^{\prime}\right)$ are neighbourhoods of $a$. Let $l$ be the line determined by $z_{0}$ and $z_{1}$. Let $H_{1}$ and $H_{2}$ be the open half planes determined by $l$ and let $N_{i}=H_{i} \cap N, N_{i}^{\prime}=H_{i} \cap N^{\prime}(i=1,2)$. Since $f\left(N_{1}\right), f\left(N_{1}^{\prime}\right)$ are both open, since $\overline{f\left(N_{1}\right)}$ contains a neighbourhood of $a$ and since $a \in \overline{f\left(N_{1}^{\prime}\right)}$, it follows that $f\left(N_{1}\right) \cap f\left(N_{1}^{\prime}\right)$ is a non empty open set with $a \in \overline{f\left(N_{1}\right) \cap f\left(N_{1}^{\prime}\right)}$. Thus we can find sequences $\left\{\xi_{k}\right\} \subseteq N_{1}$ and $\left\{\eta_{k}\right\} \subseteq N_{1}^{\prime}$ with the following properties:

(i) $\lim _{k \rightarrow \infty} \xi_{k}=z_{0}$ and $\lim _{k \rightarrow \infty} \eta_{k}=z_{1}$

(ii) $f\left(\xi_{k}\right)=f\left(\eta_{k}\right)(k=1,2, \ldots)$.

Since $N_{1}$ and $N_{2}$ are open, we may assume, by a subsequence argument that the segments $\left\{\left[\xi_{k}, \eta_{k}\right]\right\}$ are pairwise disjoint. By (ii) and RP, there is a point $\rho_{k} \in\left(\xi_{k}, \eta_{k}\right)$ with $f^{\prime}\left(\rho_{k}\right)=0 \quad(k=1,2, \ldots)$. Since the segments $\left\{\left[\xi_{k}, \eta_{k}\right]\right\}$ are disjoint, the $\rho_{k}$ 's produced are distinct. By another subsequence argument we may assume $\lim _{k \rightarrow \infty} \rho_{k}=\rho$ exists where $\rho \in\left[z_{0}, z_{1}\right] \subseteq D$. But then the Identity Theorem [4, p. 209] implies $f^{\prime}(z) \equiv 0$ on $D$, contradicting the assumption that $f$ is not identically constant. Thus Case 2 cannot occur.

The argument that Case 3 cannot occur is similar and is left to the reader.

I wish to thank the referee for many useful suggestions.

\section{REFERENCES}

1. R. M. McLeod, Mean value theorems for vector valued functions, Proc. Edinburgh Math. Soc. (2) 14 (1965), 197-209.

2. W. P. NovingeR, A local mean value theorem for analytic functions with smooth boundary values, Glasgow Math. J. 15 (1974), 27-29. 
3. J. M. Robertson, A local mean value theorem for the complex plane, Proc. Edinburgh Math. Soc. (2) 16 (1968/69), 329-331.

4. Walter Rudin, Real and Complex Analysis, (McGraw-Hill, New York, 1966).

5. Áke Samuelson, A local mean value theorem for analytic functions, American Math. Monthly 80 (1973), 45-46.

IOWA STATE UNIVERSITY

Ames, Iowa 50010 